\title{
A new method for the radiological assessment of vertebral squaring in ankylosing spondylitis
}

\author{
Stuart H Ralston, Grant D K Urquhart, Maciej Brzeski, Roger D Sturrock
}

\begin{abstract}
A method has been developed for the objective assessment of vertebral 'squaring' based on quantitative morphometric analysis of vertebral 'concavity' in lateral radiographs of the lumbar spine.

The reference range for vertebral concavity was defined as $>1.0-4.0 \mathrm{~mm}$ from measurements of 255 radiologically normal lumbar vertebrae in 51 patients with non-specific back pain. Evidence of vertebral squaring, as defined by concavity measurements of $1 \mathrm{~mm}$ or less, was found in $28 \%$ of vertebrae from 103 patients with ankylosing spondylitis and $8 \%$ of vertebrae from 10 patients with Reiter's syndrome.

Assessment of vertebral squaring by the concavity measurement was more reproducible than subjective analysis in the diagnosis of vertebral squaring; in a subgroup of 30 patients with seronegative spondyloarthropathy, the interobserver agreement on the presence or absence of vertebral squaring as assessed by two independent clinicians was $84 \%$, compared with $94 \%$ using the concavity measurement. Corresponding values for intraobserver agreement were $79 \%$ for subjective analysis and $\mathbf{8 4 \%}$ for the concavity measurement.

The vertebral concavity measurement is performed simply, rapidly (about three minutes for five lumbar vertebrae), and requires no special experience or equipment. As a result of this, the technique may be of value in the radiological diagnosis of ankylosing spondylitis and in assessing changes in vertebral squaring in individual patients with time.
\end{abstract}

Vertebral 'squaring' is a well recognised radiological feature of ankylosing spondylitis, which is typically apparent on lateral radiographs of the lumbar spine. ${ }^{1}$ The 'square' appearance is thought to result from erosion of bone at the site of ligamentous attachments to the vertebral body, but may also occur as the result of new bone formation on the anterior aspect of the vertebral body. ${ }^{1-3}$

At present, the definition of vertebral squaring is empirical and largely based on the subjective impression of the examining clinician. As vertebral squaring may be of diagnostic value in̈ankylosing spondylitis, ${ }^{1}$ a more objective method of assessment would be helpful, both diagnostically and in recording the progression of changes with time. In this study we have devised such a method, based on quantitative assessment of vertebral morphology in lateral radiographs of the lumbar spine.
Patients and methods

The study group consisted of 113 consecutive patients with seronegative spondyloarthropathies and 51 patients with a history of nonspecific back pain. Of the patients with spondyloarthropathy, 103 had been diagnosed as having ankylosing spondylitis on the basis of the New York criteria, ${ }^{4}$ and 10 had Reiter's syndrome $e^{5}$ without clinical evidence of spinal abnormalities. Patients with non-specific back pain were diagnosed on the basis of normal spinal and sacro-iliac radiographs, normal results on haematological and biochemical screening, and a negative physical examination. ${ }^{6}$

In each patient, measurements of vertebral morphology were made on lateral radiographs of the lumbar spine which had been obtained as part of the patients' routine clinical investigation.

The degree of squaring as judged by vertebral morphometry was assessed on radiographs from all patients by one observer (SHR) who was blind to the clinical details.

On the lateral radiographs of the spine, a vertical line was drawn in soft pencil, joining the upper and lower margins of each vertebral body at the junction of the vertebral end plate and anterior surface of the vertebral body. The distance between this line and the anterior aspect of the vertebral body at its most concave point was then measured to the nearest $0.5 \mathrm{~mm}$ using a transparent ruler marked with $1 \mathrm{~mm}$ gradations. This distance was referred to as the concavity measurement (fig 1). It can be appreciated that, using this method, the degree of squaring of any individual vertebrae will be inversely proportional to the concavity measurement and that a vertebra with a completely square configuration has a score of zero.

Following assessment, all pencil marks were erased from the radiographs to avoid introducing bias in the assessment of subsequent observers.

Subjective analysis of the presence or absence of squaring in individual vertebrae was made independently on the same radiographs by a radiologist (GDKU) who was blind with respect to the earlier results and the clinical details.

In some patients with advanced ankylosing spondylitis, the presence of large anteriorly placed syndesmophytes made the morphometric assessment inaccurate as a result of difficulties in defining the anterior margin of the vertebral body. Those vertebrae were excluded, resulting in a final total of 417 evaluable vertebrae out of the original total of 515 which were studied. Although the presence of Romanus lesions would, on a theoretical basis, also have precluded accurate assessment of the concavity measurement, no such lesions 
were identified in patients who were entered into this study.

Inter- and intraobserver variability were determined by analysis of 150 vertebrae from a subgroup of 30 patients with seronegative spondyloarthropathy (27 patients with ankylosing spondylitis, three patients with Reiter's syndrome), chosen at random from the study group. The intraobserver variability was based on duplicate assessments performed on separate occasions by the same observers (SHR, concavity measurements; GDKU, subjective analysis). At the time of the repeat assessment, the two observers were blind to the previous results. The interobserver variability was based on assessment of the same vertebra by two further

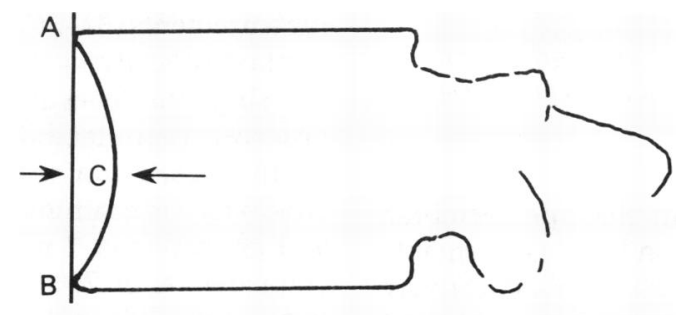

$\mathrm{C}=$ concavity score $(\mathrm{mm})$

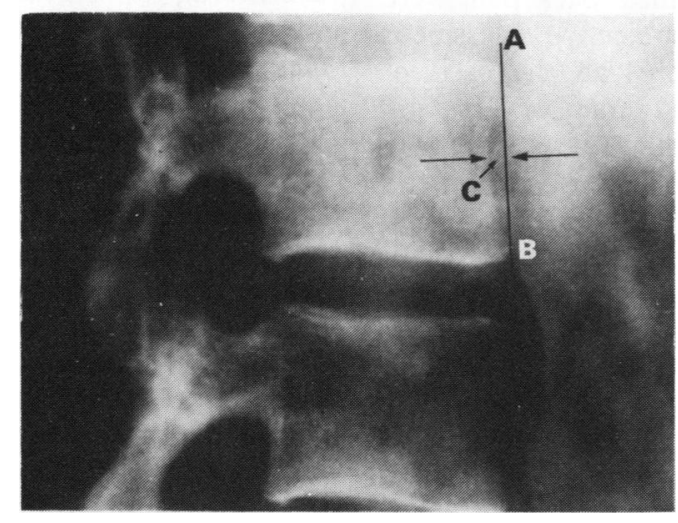

Figure 1 Method of assessing concavity measurements. The concavity measurement was derived by drawing $a$ vertical line joining the upper $(A)$ and lower $(B)$ margins of the vertebral body at the junction on the vertebral end plate and the anterior vertebral margin. The distance in millimetres between the line and the most concave aspect of the vertebral body $(C)$ was referred to as the concavity measurement.

Table 1 Clinical details and concavity scores in study group. Values for age and disease duration are means (SEM); values for concavity scores are medians (interquartile range).

\begin{tabular}{|c|c|c|c|}
\hline & \multicolumn{3}{|c|}{ Subject classification } \\
\hline & $\begin{array}{l}\text { Ankylosing } \\
\text { spondylitis } \\
(n=103)\end{array}$ & $\begin{array}{l}\text { Reiter's } \\
\text { syndrome } \\
(n=10)\end{array}$ & $\begin{array}{l}\text { Non-specific } \\
\text { back pain } \\
(n=5 I)\end{array}$ \\
\hline $\begin{array}{l}\text { No of men }(\%) \\
\text { Age (years) } \\
\text { Disease duration (years) } \\
\text { Lumbar vertebra } 1(\mathrm{~mm}) \\
\text { Lumbar vertebra } 2(\mathrm{~mm}) \\
\text { Lumbar vertebra } 3(\mathrm{~mm}) \\
\text { Lumbar vertebra } 4(\mathrm{~mm}) \\
\text { Lumbar vertebra } 5(\mathrm{~mm}) \\
\text { All lumbar vertebra }(\mathrm{mm})\end{array}$ & $\begin{array}{l}87(84) \\
40(10) \\
16(10) \\
2 \cdot 0 \\
(1 \cdot 5-3 \cdot 0) \\
2 \cdot 25 \\
(1 \cdot 5-3 \cdot 0) \\
2 \cdot 0 t \\
(1 \cdot 0-2 \cdot 5) \\
1 \cdot 5 t \\
(1 \cdot 0-2 \cdot 5) \\
1 \cdot 5 \dagger \\
(0 \cdot 5-2 \cdot 0) \\
2 \cdot 0 \\
(1 \cdot 0-2 \cdot 5)\end{array}$ & $\begin{array}{l}8(80) \\
40(12) \\
8(7) \\
3 \cdot 0^{* * *} \\
(2 \cdot 5-4 \cdot 0) \\
3 \cdot 0^{* *} \\
(2 \cdot 5-4 \cdot 0) \\
2 \cdot 75^{*} \\
(1 \cdot 5-3 \cdot 0) \\
2 \cdot 0 \\
(1 \cdot 25-3 \cdot 25) \\
2 \cdot 5^{* * *} \\
(2 \cdot 0-4 \cdot 0) \\
3 \cdot 0 * * * \\
(2 \cdot 0-3 \cdot 5)\end{array}$ & $\begin{array}{l}29(58) \\
37(10) \ddagger \\
\text { NR } \\
3 \cdot 0^{* *} \\
(2 \cdot 5-3 \cdot 5) \\
2 \cdot 5 \dagger \\
(2 \cdot 0-3 \cdot 0) \\
2 \cdot 0 \dagger \\
(1 \cdot 5-2 \cdot 5) \\
2 \cdot 0^{* * * \dagger} \\
(2 \cdot 0-2 \cdot 5) \\
3 \cdot 0^{* * *} \\
(2 \cdot 0-3 \cdot 5) \\
2 \cdot 5^{* * *} \\
(2 \cdot 0-3 \cdot 0)\end{array}$ \\
\hline
\end{tabular}

NR $=$ not recorded.

${ }^{*} p<0.05 ;{ }^{*} p<0.01 ; * * *<<0.001$ from ankylosing spondylitis group.

tp<0.01 from vertebra 1 within groups.

$\neq \mathrm{p}<0.001$ from non-specific back pain group. observers (MB, concavity measurement; RDS, subjective assessment) who were blind with respect to the other observers' results.

In exploratory analysis, the degree of vertebral squaring as assessed by the concavity measurement was also expressed as a ratio to the anterior vertebral height, measured in millimetres on lateral radiographs. The correlation between this ratio and the concavity measurement approached unity, however $(r=0.989$; $\mathrm{p}<0.0001$ ), and the direct measurement alone was therefore used throughout the study.

Statistical tests used in analysis were the Mann-Whitney test, Spearman rank correlation coefficient, linear regression analysis, and the $\chi^{2}$ test.

\section{Results}

Table 1 gives the relevant clinical data and concavity measurements from the three patient groups. Overall, vertebrae from patients with ankylosing spondylitis had lower concavity measurements than those from patients with non-specific back pain and Reiter's syndrome, though when individual vertebrae were assessed separately, the differences between the groups were not statistically significant for lumbar vertebrae 2 and 3 (ankylosing spondylitis $v$ nonspecific back pain) and 4 (ankylosing spondylitis $v$ Reiter's syndrome).

In all three groups, the median concavity measurement was higher in lumbar vertebra 1 than in lumbar vertebrae 3 and 4, though the differences between vertebrae were only statistically significant in the groups with ankylosing spondylitis and non-specific back pain.

Patients with mechanical back pain were slightly younger than patients with ankylosing spondylitis and the proportion of women was higher. These factors were unlikely to have explained the other differences between the groups, however, as there was no correlation between age and concavity measurements in the non-specific back pain group $(r=-0 \cdot 12$, not significant (NS)) and no difference between concavity measurements in women (median score 2.5 ) and men (median score 2.5).

Figure 2 shows the distribution of concavity measurements in the three groups in relation to the subjective assessment of squaring by a radiologist. In patients with non-specific back pain and Reiter's syndrome the concavity measurements followed a Gaussian distribution, whereas the distribution in patients with ankylosing spondylitis was bimodal, with an initial peak at $0 \mathrm{~mm}$ and a further peak at $2.0 \mathrm{~mm}$. A reference range for vertebral concavity was defined as $0.87-4.21 \mathrm{~mm}$ on the basis of the mean (2SD) values in patients with mechanical back pain. As the measurements were in practice made to the nearest $0.5 \mathrm{~mm}$, and values in the group with non-specific back pain were skewed towards lower values, the reference range was taken as $>1.0-4.0 \mathrm{~mm}$. Although the median concavity measurements differed slightly between vertebrae (table 1), there was no significant difference in the lower limit of vertebral concavity between different vertebrae (data not shown). 


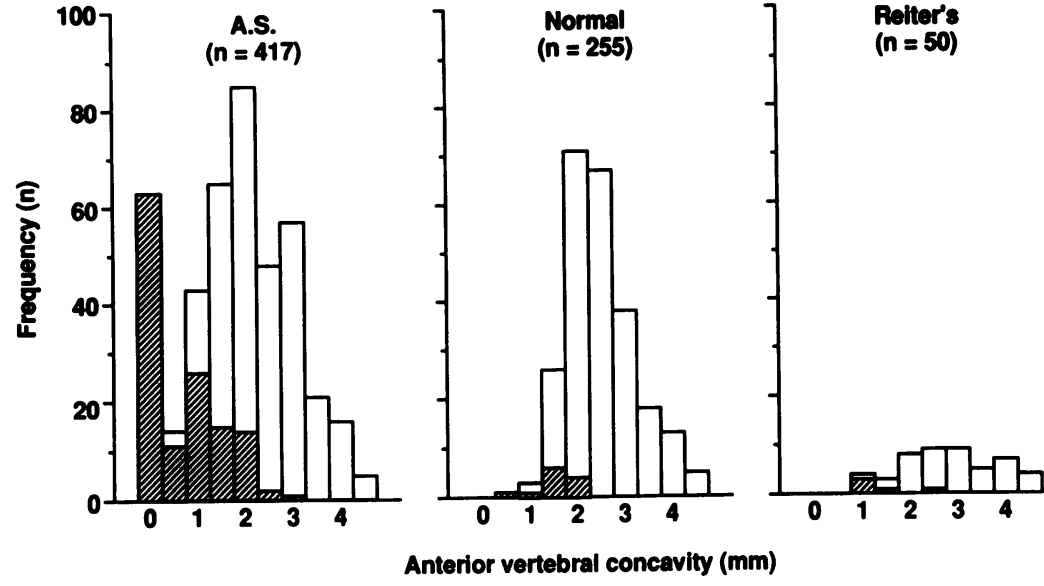

Figure 2 Distribution of concavity measurements in study group. The columns represent the total number of vertebrae in each group in relation to the measured vertebral concavity as described in fig 1 . The hatched parts of the columns represent the number of vertebrae in each group which were considered square by a radiologist, who read the films blind without knowledge of the concavity measurement.

From fig 1 it is apparent that the vertebrae which had the lowest concavity measurements also tended to be those which were subjectively considered square by the radiologist (shaded columns). Thus, $12 / 255(4 \cdot 7 \%)$ of the vertebrae from patients with mechanical back pain were thought to be square on subjective assessment compared with $4 / 255(1.5 \%)$ on the basis of concavity measurements $1 \mathrm{~mm}$ or less $\left(\chi^{2}\right.$ $4 \cdot 13 ; p<0.05)$. Corresponding values were $6 / 55$ (7.2\%), compared with $2 / 55$ (3.6\%) in Reiter's syndrome $\left(\chi^{2} 2 \cdot 15, \mathrm{NS}\right)$ and $132 / 417(31 \%)$ compared with $119 / 417(28 \%)$ in patients with ankylosing spondylitis $\left(\chi^{2} 0.96\right.$, NS). There was agreement between the presence or absence of squaring as subjectively assessed by a radiologist (GDKU) and objectively assessed by concavity measurements $1 \mathrm{~mm}$ or less in $365 / 417(87 \%)$ of vertebrae in patients with ankylosing spondylitis, $243 / 255(95 \%)$ of vertebrae in patients with non-specific back pain, and $46 / 50(92 \%)$ of vertebrae in patients with Reiter's syndrome.

Table 2 gives details of intraobserver and interobserver reproducibility for the subjective and objective methods of assessing vertebral squaring. On subjective assessment, observer 2 tended to over diagnose vertebral squaring (56\% of subjects) compared with observer 1

Table 2 Intraobserver and interobserver reproducibility for assessment of vertebral squaring. Values given as number (\%)

\begin{tabular}{lll}
\hline & $\begin{array}{l}\text { Square } \\
\text { vertebrae }\end{array}$ & $\begin{array}{l}\text { Agreement } \\
\text { between } \\
\text { assessments }\end{array}$ \\
\hline $\begin{array}{c}\text { Subjective analysis } \\
\text { Observer 1.1 } \\
\text { (GDKU) } \\
\text { Observer } 1 \cdot 2 \\
\text { (GDKU) }\end{array}$ & $65 / 150(43)$ \\
$\begin{array}{c}\text { Observer } 2 \\
\text { (RDS) }\end{array}$ & $60 / 150(40)$ \\
$\begin{array}{c}\text { Objective analysis (concavity measurement) } \\
\text { Observer } 3 \cdot 1 \\
\text { (SHR) }\end{array}$ & $55 / 150(56)$ \\
$\begin{array}{c}\text { Observer } 3 \cdot 2 \\
\text { (SHR) }\end{array}$ & $55 / 150(36)^{*}$ \\
$\begin{array}{c}\text { Observer } 4 \\
\text { (MB) }\end{array}$ & $47 / 150(36)^{*}$ \\
\hline
\end{tabular}

*Vertebrae with concavity scores of $<1 \cdot 0 \mathrm{~mm}$.
( $40 \%$ of subjects), with an overall interobserver agreement of $79 \%$. Observer 1 recognised similar total numbers of square vertebrae between assessments $(\mathbf{4 0} \%$ of subjects compared with $43 \%$ ), but the actual intraobserver agreement between the assessments was $84 \%$, as some vertebrae were considered square on the first assessment but not on the second, and vice versa.

On objective assessment, there was a close correlation between the concavity measurements made by observer 3 and observer $4(r=0.92$; $p<0.001)$ and between the two assessments made by observer 3 on separate occasions $(\mathrm{r}=0.94 ; \mathrm{p}<0.001)$ (individual data points not shown). The two observers recorded fewer square vertebrae on the basis of concavity measurements (31-36\%) than with the subjective method of analysis (40-56\%) (table 2).

Using the concavity measurement, interobserver reproducibility between assessments for the presence or absence of squaring - that is, concavity measurements of $1 \mathrm{~mm}$ or less and greater than $1 \mathrm{~mm}$ respectively-was $92 \%$ compared with $79 \%$ for the subjective assessment $\left(\chi^{2} 7.8 ; p<0.01\right)$. The intraobserver reproducibility of the concavity measurement in confirming or excluding squaring was $94 \%$ compared with $84 \%$ for the subjective assessment $\left(\chi^{2} 14.6 ; p<0.001\right)$.

\section{Discussion}

We believe that the concavity score devised in this study has several advantages over subjective techniques in the assessment of vertebral squaring. The concavity measurement can be performed simply and rapidly on routine lateral radiographs of the lumbar spine and requires no special experience or equipment-the average time to assess all five lumbar vertebrae was less than three minutes. Its main advantage over the traditional method of assessment is that it is a quantifiable, relatively objective measure which is independent of the previous experience of the observer. It is, in addition, more reproducible than subjective assessment and in this respect may be of value in recording changes in the degree of vertebral squaring over time. Its only limitation is in patients with anterior syndesmophyte formation, where the measurements cannot be made easily as a result of difficulty in defining the anterior margin of the vertebral body, though this limitation also applies to subjective assessment of vertebral squaring.

When the technique was applied to patients with ankylosing spondylitis, the overall incidence of vertebral squaring was $28 \%$, compared with $31 \%$ on subjective assessment by a radiologist. These figures are substantially lower than that reported by Spencer et al, ${ }^{7}$ who found evidence of vertebral squaring in $73.5 \%$ of 200 patients with ankylosing spondylitis. These differences may partly be a result of differences in patient selection, absence of an objective method for measuring squaring, and inclusion of thoracic vertebrae in the earlier study, as it is known that squaring may often be seen in the thoracic vertebrae of normal subjects. $^{1}$ 
Further studies will be necessary to define the role of the concavity measurements in clinical practice, though potential applications include the early diagnosis of ankylosing spondylitis and monitoring changes with time in response to potential disease modifying agents.

1 Resnick D, Niwayama G. Ankylosing spondylitis. In: Resnick D, Niwayama G, eds. Diagnosis of bone and joint disorders. Vol. 2. Philadelphia: Saunders, 1987: 1040-1102.

2 Aufdermann M. Pathogenesis of square bodies in ankylosing spondylitis. Ann Rheum Dis 1989; 48: 628-32.
3 Calin A. Ankylosing spondylitis. In: Kelley W N, Harris E D, Ruddy S, Sledge C B, eds. Textbook of Rheumatology. Vol. 2. 3rd ed. Philadelphia: Saunders, 1989: 1021-38.

4 Moll J M H, Wright V. New York clinical criteria for an ankylosing spondylitis. Ann Rheum Dis 1973; 32: 354-63.

5 Calin A. Reiter's syndrome. In: Kelley W N, Harris E D, Ruddy S, Sledge C B, eds. Textbook of Rheumatology. Vol. 2. 3rd ed. Philadelphia: Saunders, 1980: 1038-52.

6 Dixon A St J. Diagnosis of low back pain - sorting out the complainers. In: Jayson M I V, ed. The lumbar spine and back pain. 2nd ed. Tunbridge Wells: Pitman, 1980: back pain.

7 Spencer D G, Park W M, Dick H M, Papazoglou S N, Buchanan WW. Radiological manifestations in 200 patients with ankylosing spondylitis: correlation with clinical features and HLA B27. I R heumatol 1979; 6: 305-15. 